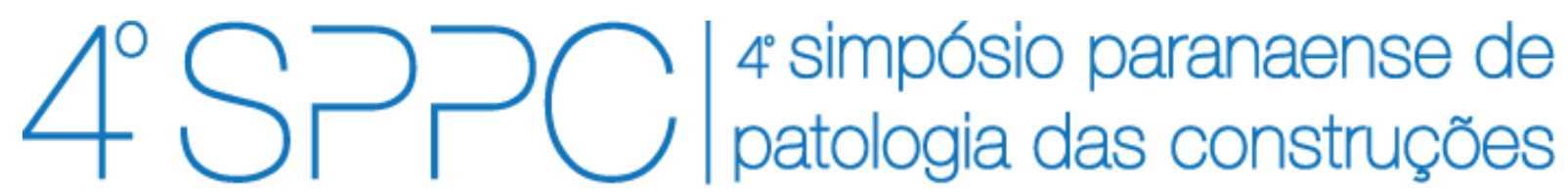

ISSN 2526-7248 artigo n. 4SPPC109, pp. 70-79, 2019

\title{
Avaliação da penetração acelerada de íons cloreto em concretos produzidos com materiais da cidade de Fortaleza
}

\author{
Dias, Alisson Rodrigues de Oliveira ${ }^{1}$; Amancio, Felipe Alves ${ }^{1}$; Lucas, Sarah Oliveira ${ }^{2}$ \\ ; Lima, Douglas Alexandre²; Cabral, Antônio Eduardo Bezerra3; \\ ${ }^{1}$ Mestre em Engenharia Civil, Universidade Federal do Ceará, \\ rodrigues_alisson@live.com, felipeaamancio@hotmail.com \\ ${ }^{2}$ Graduando em Engenharia Civil, Universidade Federal do Ceará, \\ saraholiveiraluks@gmail.com, douglasalexandre@alu.ufc.br \\ ${ }^{3}$ Doutor em Engenharia Civil, Universidade Federal do Ceará, \\ eduardo.cabral@ufc.br
}

Resumo: Dentre as inúmeras manifestações patológicas que afetam as estruturas de concreto armado, têm-se grande destaque, a corrosão. Tal deterioração pode ser causada por diversos agentes, entre eles têm-se a penetração de íons cloreto, em virtude da agressividade do ar em regiões marítimas, consequência da elevada concentração desses íons nas névoas salinas das cidades litorâneas. Neste sentido, foram produzidos três traços de concreto nas classes de resistência de 20, 30 e 40 $\mathrm{MPa}$ e caracterizadas as propriedades no estado endurecido como: resistência à compressão, absorção de água, índice de vazios e penetração acelerada de íons cloretos conforme a ASTM C1202. Os resultados demonstraram que os concretos de fck 20, 30 e 40MPa apresentaram risco de penetração de íons cloreto em alto, moderado e baixo, respectivamente. Isto é, concretos com maiores fck apresentaram os menores valores de carga passante. Isto se deve ao menor fator $\mathrm{a} / \mathrm{c}$ desses concretos, e consequentemente, a menor porosidade, colaborando para o aumento de sua durabilidade.

Palavras-chave: Concreto, Penetração de íons cloreto, ASTM C1202, durabilidade.

Abstract: Among the innumerable pathological manifestations that affect the structures of reinforced concrete, a great prominence is the corrosion. Such deterioration can be caused by several agents, among them are the chloride ions penetration, due to the aggressiveness of the air in the maritime regions, consequence of the high concentration of chloride ions in the saline mists of coastal cities. In this sense, three mixes of concrete were produced in the strength classes of 20, 30 and $40 \mathrm{MPa}$ and characterized the properties in hardened state as: compressive strength, water absorption, voids index and accelerated chlorides ions penetration according to ASTM C1202. The results demonstrated that concrete of fck 20, 30 and $40 \mathrm{MPa}$ presented a risk of chloride ions penetration in high, moderate and low, respectively. That is, concrete with higher fck presented lower values of charge passed. This is due to the lower w/c ratio of these concretes, and consequently, lower porosity, which increases its durability.

Keywords: Concrete, Penetration of chloride ions, ASTM C1202, Durability. 
DIAS, A. R. O.; AMANCIO, F. A.; LUCAS, S. O.; LIMA, D. A.; CABRAL, A. E. B., AVALIAÇÃO DA PENETRAÇÃO ACELERADA DE ÍNS CLORETOS EM CONCRETOS PRODUZIDOS COM MATERIAIS DA CIDADE DE FORTALEZA. $4^{\circ}$ Simpósio Paranaense de Patologia das Construções (40 SPPC), artigo 4SPPC109, pp. 70 - 79, 2019. DOI: 10.4322/2526-7248.030

\section{Introdução}

O concreto é o segundo material mais consumido no mundo, atrás somente da água [1]. Antigamente, acreditava-se que a vida útil do concreto armado era ilimitada. Entretanto, a deteriorização precoce destas estruturas ocasionadas pela corrosão vem crescendo consideravelmente [2].

Dentre as diversas manifestações patológicas que afetam as estruturas de concreto, têm-se grande destaque a corrosão. Estima-se que são gastos mundialmente, bilhões de dólares em recuperações e reparos de estruturas de concreto armado, devido a corrosão, o que acaba por afetar sua estabilidade e funcionalidade [3].

Em geral, esta corrosão é causada pelo ataque destrutivo dos íons cloreto que penetram por difusão e/ou outros mecanismos de penetração a partir do exterior [2]. Tal ataque acaba por ocasionar à redução da resistência, facilidade de manutenção e estética da estrutura [4]. Os cloretos são prejudiciais ao concreto armado, pois com a chegada destes íons à armadura de aço, ocorre uma fragmentação do concreto, o que acaba por gerar a entrada de novos íons cloretos aos níveis subsequentes da armadura [5].

A corrosão das estruturas de concreto armado na maioria das vezes se deve a ação da carbonatação. Entretanto, nas regiões marítimas, os cloretos contidos na névoa salina, se constituem a causa mais significativadas manifestações patológica das estruturas de concreto armado expostas nesse ambiente [6,7].

Conforme estudo realizado por Campos [8] verificou-se a alta agressividade do ar atmosférico da cidade de Fortaleza, no que diz respeito a concentração de íons cloreto. Verificou-se ainda que na cidade, o comportamento da agressividade é diferente, primordialmente em função da direção dos ventos, tendo alguns locais, como a praia do Futuro, concentrações de íons cloro foram superiores a de outras cidades brasileiras litorâneas.

Em adição, ressalta-se que a baixa qualidade do concreto empregado nas obras, que pode ser devido a erro de dosagem, processo de transporte, adensamento e cura deficientes o que pode ocasionar elevada porosidade, segregações e entre outras, acabam por facilitar o processo de iniciação da corrosão das estruturas [3].

O método de ensaio para avaliar a penetração de íons cloreto é preconizado pela ASTM C1202:2012 [9], e apresenta vantagens em virtude de ser um método de simples execução, rapidez na obtenção dos dados e aplicável a quase todos os tipos de concreto, principalmente no intuito de comparar os resultados entre concretos com traços distintos, com um de referência [10]

Diante do exposto, é de extrema importância estudos no intuito de investigar a durabilidade deste material, conhecendo os fatores e suas causas. Neste sentido, o presente trabalho visa investigar a resistência a penetração acelerada de íons de cloreto em concretos produzidos na cidade de Fortaleza-CE. 
DIAS, A. R. O.; AMANCIO, F. A.; LUCAS, S. O.; LIMA, D. A.; CABRAL, A. E. B., AVALIAÇÃO DA PENETRAÇÃO ACELERADA DE ÍONS CLORETOS EM CONCRETOS PRODUZIDOS COM MATERIAIS DA CIDADE DE FORTALEZA. $4^{\circ}$ Simpósio Paranaense de Patologia das Construções (40 SPPC), artigo 4SPPC109, pp. 70 - 79, 2019. DOI: 10.4322/2526-7248.030

\section{Materiais e métodos}

\subsection{Materiais}

Nesta pesquisa foi utilizado cimento Portland CP V - ARI. As Tabelas 1 e 2 apresentam as características físicas e químicas do cimento fornecidas pelo fabricante.

Tabela 1 - Características físicas e químicas do cimento utilizado

\begin{tabular}{cccc}
\hline Ensaio & Norma & Resultado & $\begin{array}{c}\text { Especificação } \\
\text { NBR 16697/2018 }\end{array}$ \\
\hline Perda ao fogo & NM 18/12 & $4,39 \%$ & $\leq 6,5$ \\
Óxido de Magnésio - MgO & NM 11-2/12 & $4,17 \%$ & $\leq 6,5$ \\
Anidrido Sulfúrico - $\mathrm{SO}_{3}$ & $\mathrm{NM} \mathrm{16/12}$ & $3,24 \%$ & $\leq 4,5$ \\
Resíduo Insolúvel & $\mathrm{NM} \mathrm{15/12}$ & $0,69 \%$ & $\leq 3,5$ \\
Anidrido Carbônico - $\mathrm{CO}_{2}$ & $11578 / 91$ & $2,97 \%$ & $\leq 5,5$ \\
Massa específica & $\mathrm{NM} \mathrm{23/01}$ & $3,06 \mathrm{~g} / \mathrm{cm}^{3}$ & $\leq 6,0$ \\
Finura (\#325) & $12826 / 14$ & $4,8 \%$ & $\geq 1: 00 \mathrm{~h}: \mathrm{min}$ \\
Início de pega & NM 65/03 & $2: 15 \mathrm{~h}: \mathrm{min}$ & $\leq 10: 00 \mathrm{~h}: \mathrm{min}$ \\
Fim de pega & NM 65/03 & $3: 45 \mathrm{~h}: \mathrm{min}$ & $\leq 5: 00 \mathrm{~h}: \mathrm{min}$ \\
Expansibilidade a quente & $11582 / 16$ & $0,00 \mathrm{~mm}$ & $\leq 6,5$ \\
\hline
\end{tabular}

Tabela 2: Resistência à compressão em MPa do cimento utilizado

\begin{tabular}{ccc}
\hline Idade (dias) & Média (MPa) & $\begin{array}{c}\text { Especificação } \\
\text { NBR 16697/2018 }\end{array}$ \\
\hline 1 & 25,9 & $\geq 14,0 \mathrm{Mpa}$ \\
3 & 36,6 & $\geq 24,0 \mathrm{Mpa}$ \\
7 & 44,3 & $\geq 34,0 \mathrm{Mpa}$ \\
\hline
\end{tabular}

A água utilizada atendeu os requisitos da ABNT NBR 15900-1:2009 [11]. O aditivo foi do tipo superplastificante da linha MasterGlenium ACE 402, composto por carboxilatos, a base de naftaleno sulfonato. $\mathrm{O}$ aditivo possui possui $\mathrm{pH}$ de 7 , densidade de $1,15 \mathrm{~g} / \mathrm{cm}^{3}$ e solubilidade em agua de $100 \mathrm{~g} / \mathrm{L}$.

Os agregados miúdos e graúdos utilizados foram provenientes da Região Metropolitana de Fortaleza, atendendo a ABNT NBR 7211:2009 [12]. A Tabela 3 apresenta as propriedades físicas dos agregados utilizados. A granulometria dos agregados miúdos é apresentada na Figura 1.

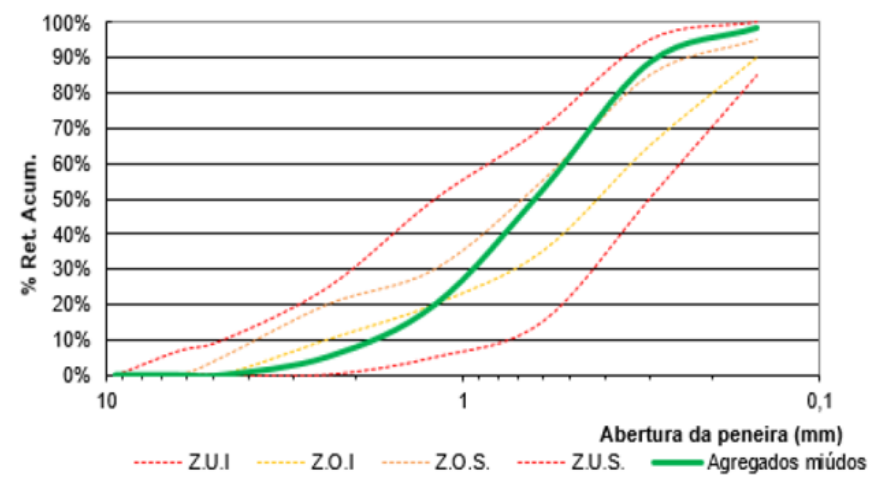

Figura 1: Curva granulométrica do agregado miúdo 
Tabela 3: Propriedades fisicas dos agregados utilizados

\begin{tabular}{ccccc}
\hline & & \multicolumn{3}{c}{ Agregado } \\
\cline { 3 - 5 } Ensaio & Unid. & Areia & $\begin{array}{c}\text { Brita } \\
\mathbf{1 2 , 5} \mathbf{~ m m}\end{array}$ & $\begin{array}{c}\text { Brita } \\
\mathbf{1 9 m}\end{array}$ \\
\hline Diâmetro máximo caracteristico & $\mathrm{mm}$ & 4,75 & 12,5 & 19 \\
Módulo de finura & Adim & 2,65 & 5,99 & 6,84 \\
Massa unitária & $\mathrm{g} / \mathrm{cm}^{3}$ & 1,41 & 1,41 & 1,37 \\
Absorção de água & $\%$ & 0,9 & 0,89 & 0,94 \\
Massa específica & $\mathrm{g} / \mathrm{cm}^{3}$ & 2,59 & 2,61 & 2,61 \\
Material pulverulento & $\%$ & 1,0 & 0,29 & 0,9 \\
\hline
\end{tabular}

\subsection{Métodos de pesquisa}

Os traços adotados foram usuais da cidade de Fortaleza, Ceará. Os traços possuem classes de resistência C20, C30 e C40, ou seja, devendo atingir aos 28 dias as resistências características de 20, 30 e $40 \mathrm{MPa}$, respectivamente.

A consistência no estado fresco foi fixada em $100 \pm 10 \mathrm{~mm}$, por meio do slump test, conforme ABNT NBR NM 67:1998 [13]. A Tabela 4 apresenta o consumo de materiais dos traços investigados, em $\mathrm{kg} / \mathrm{m}^{3}$.

Tabela 4: Consumo de materiais $\mathrm{em} \mathrm{kg} / \mathrm{m}^{3}$ para os traços investigados

\begin{tabular}{ccccccc}
\hline Classe & Cimento & Areia & $\begin{array}{c}\text { Brita } \\
\mathbf{1 2 , 5 m m}\end{array}$ & $\begin{array}{c}\text { Brita } \\
\mathbf{1 9} \mathbf{~ m m}\end{array}$ & $\begin{array}{c}\text { Aditivo } \\
\text { (\%) }\end{array}$ & a/c \\
\hline C20 & 260 & 860 & 277 & 694 & 0,37 & 0,69 \\
C30 & 360 & 794 & 264 & 703 & 0,28 & 0,50 \\
C40 & 440 & 710 & 266 & 709 & 0,33 & 0,41 \\
\hline
\end{tabular}

No estado fresco, após a determinação da trabalhabilidade por meio do ensaio slump test, deu-se início a moldagem dos corpos de prova de dimensão $10 \times 20 \mathrm{~cm}$ (diâmetro e altura) e cura dos mesmos em tanques com água à temperatura ambiente. Em seguida realizou-se os ensaios de caracterização dos concretos no estado endurecido aos 28 dias, tendo sido utilizado 3 corpos de prova para cada ensaio.

A resistência à compressão foi realizada conforme preconiza a ABNT NBR 5739:2018 [14]. O módulo de elasticidade estático foi analisado para a idade de 28 dias, com a utilização de 3 corpos de prova para cada traço. Este ensaio foi realizado conforme preconiza a ABNT NBR 8522:2017 [15]. Em relação aos ensaios de durabilidade, foram realizados os ensaios de absorção de água por imersão e índice de vazios (ABNT NBR 9778:2009) [16] e determinação da capacidade de penetração acelerada de íons cloretos (ASTM C1202:2002) [9]. A Figura 2 apresenta a máquina utilizada para a realização do ensaio de penetração de cloretos. 


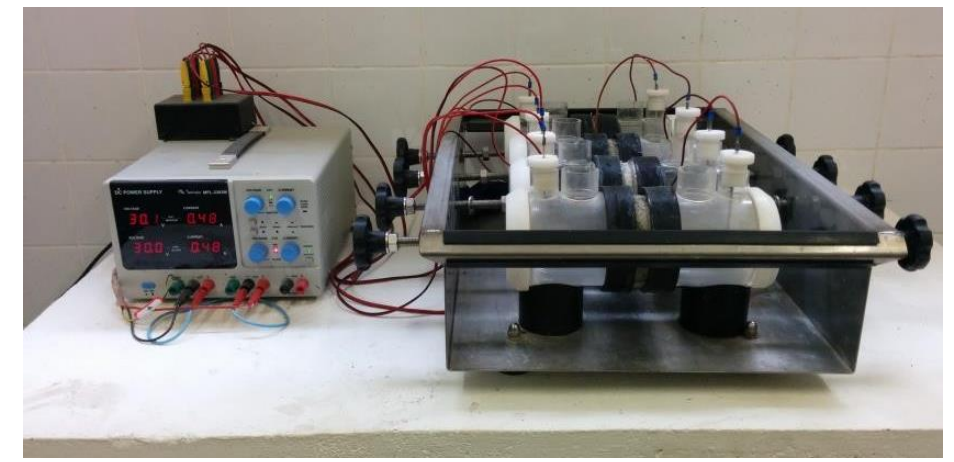

Figura 2: Aparelhagem para o ensaio de penetração acelerada de íons cloretos.

O ensaio de avaliação da penetração acelerada de íons cloretos foi conduzido de acordo com a ASTM C 1202 (2012) - Electrical Indication of Concrete's Ability to Resist Chloride Ion Penetration [9]. O método consiste em acoplar um corpo de prova cilíndrico de dimensões 10 × $5 \mathrm{~cm}$ (diâmetro e altura) entre duas meia-células acrílicas, sendo uma preenchida com uma solução aquosa de cloreto de sódio $(\mathrm{NaCl})$ com 3\% de concentração e outra com solução aquosa de hidróxido de sódio ( $\mathrm{NaOH})$ com concentração de $0,3 \mathrm{~N}$. A avaliação da penetração de íons cloretos é feita mediante a medição da carga passante na amostra de concreto, submetida a uma tensão de $60 \pm 0,1 \mathrm{~V}$ durante um período de 6 horas. Entre as meias-células é gerada uma corrente elétrica que induz o ânion cloreto a se difundir através do concreto sob a ação de um campo elétrico. A leitura da corrente passante é registrada por um amperímetro, em intervalos de trinta minutos. Conforme é ilustrado na Figura 3.

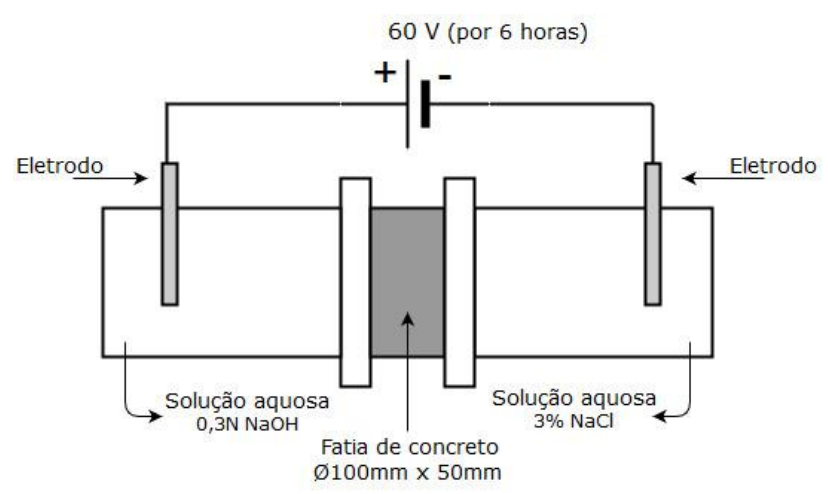

Figura 3: llustração do ensaio de penetração acelerada de íons cloretos.

Para o estudo foram moldados dois corpos de prova de dimensões $10 \times 20 \mathrm{~cm}$ para cada traço e posteriormente serrados em quatro partes perpendiculares ao eixo com dimensões de $10 \times 5 \mathrm{~cm}$ (diâmetro e altura) cada. É importante salientar que foram utilizadas as partes centrais dos corpos de prova, sendo descartadas as partes superiores e inferiores, de modo a desconsiderar o efeito de qualquer segregação e/ou exsudação que pudesse alterar os resultados. Sendo avaliados 4 amostras por traço. A carga total passante foi obtida por meio da Equação 1, sendo $Q$ - carga total passante (coulombs, C); 10 - corrente (ampères) medida imediatamente após a aplicação da tensão; e lt - corrente (amperes) medida nos intervalos "t" em minutos.

$$
Q=900 \cdot\left(I_{0}+2 \cdot I_{30}+2 \cdot I_{60}+\cdots+2 \cdot I_{330}+I_{360}\right) \quad \text { Equação } 01
$$


DIAS, A. R. O.; AMANCIO, F. A.; LUCAS, S. O.; LIMA, D. A.; CABRAL, A. E. B., AVALIAÇÃO DA PENETRAÇÃO ACELERADA DE ÍONS CLORETOS EM CONCRETOS PRODUZIDOS COM MATERIAIS DA CIDADE DE FORTALEZA. $4^{\circ}$ Simpósio Paranaense de Patologia das Construções (40 SPPC), artigo 4SPPC109, pp. 70 - 79, 2019. DOI: 10.4322/2526-7248.030

A partir do resultado obtido na Equação 1, foi possível classificar qualitativamente 0 concreto quanto ao risco de penetração de íons cloretos, conforme a Tabela 5.

Tabela 5: Classificação da penetração de íons cloretos no concreto

\section{Carga passante (C) Risco de penetração de íons cloretos}

\begin{tabular}{cc}
\hline$>4000$ & Alto \\
\hline 2000 a 4000 & Moderado \\
\hline 1000 a 2000 & Baixo \\
\hline 100 a 1000 & Muito baixo \\
\hline$<100$ & Desprezível \\
\hline
\end{tabular}

Fonte: ASTM C1202 [9].

Por fim, a análise dos dados foi realizada uma análise estatística por meio da Análise de Variância (ANOVA). Para esta análise, foi utilizado o software Statistica ${ }^{\circledR}$ 7.0. Primeiramente, obteve-se os resíduos padronizados para os ensaios realizados, no intuito de eliminar os dados espúrios. Tais valores são considerados outliers quando os resíduos padronizados excedem um valor de referência de 1,96 para mais ou para menos (valor crítico para um nível de confiança de 0,05). Após a identificação e descarte dos dados discrepantes, é realizado uma nova rodada de análise até que todos os pontos fiquem compreendidos no intervalo como aceitável. Por fim, é identificado a Significância (S) ou Não Significância(NS) dos parâmetros analisados.

\section{Resultados e Discussões}

A Tabela 6 apresenta um resumo geral dos resultados obtidos nos ensaios realizados no estado endurecido, para cada traço de concreto estudado.

Tabela 6: Caracterização dos traços de concreto no estado endurecido.

\begin{tabular}{ccccc} 
Traços & $\begin{array}{c}\text { Resist. à } \\
\text { compressão aos } \\
\text { 28 dias (MPa) }\end{array}$ & $\begin{array}{c}\text { Módulo de } \\
\text { elasticidade } \\
\text { (GPa) }\end{array}$ & $\begin{array}{c}\text { Absorção } \\
\text { imersão } \\
(\%)\end{array}$ & $\begin{array}{c}\text { Índice de } \\
\text { vazios } \\
(\%)\end{array}$ \\
\hline T20 & 20,87 & 22,18 & 3,4 & 7,43 \\
\hline T30 & 32,17 & 25,01 & 2,7 & 5,92 \\
\hline T40 & 41,4 & 30,81 & 1,7 & 3,84 \\
\hline
\end{tabular}

Destaca-se na Tabela 6 que a resistência mecânica e módulo de elasticidade são maiores nos traços de fck mais elevados, bem como os parâmetros de durabilidade, como as propriedades de absorção de água e índice de vazios, melhora com o aumento da classe de resistência. Isto se deve ao maior consumo de cimento e menor relação a/c nos traços com maiores fck.

A Figura 4 apresenta os valores de penetração acelerada de íons cloreto para os traços investigados. 
DIAS, A. R. O.; AMANCIO, F. A.; LUCAS, S. O.; LIMA, D. A.; CABRAL, A. E. B., AVALIAÇÃO DA PENETRAÇÃO ACELERADA DE ÍONS CLORETOS EM CONCRETOS PRODUZIDOS COM MATERIAIS DA CIDADE DE FORTALEZA. $4^{\circ}$ Simpósio Paranaense de Patologia das Construções (40 SPPC), artigo 4SPPC109, pp. 70 - 79, 2019. DOI: 10.4322/2526-7248.030
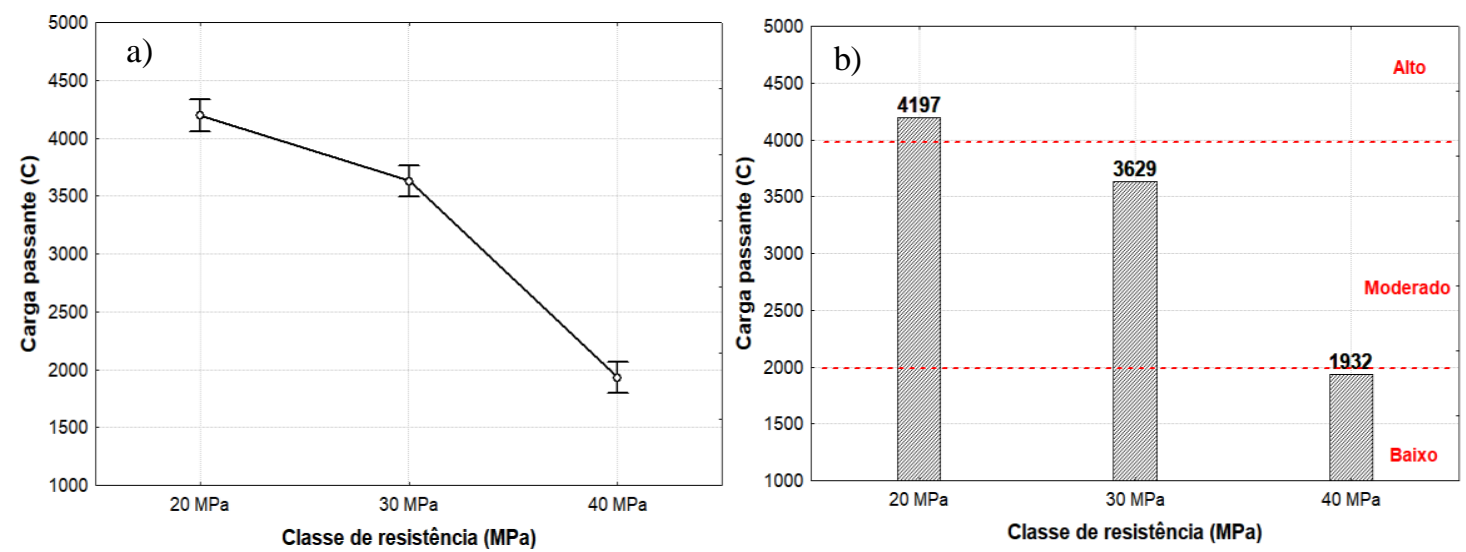

Figura 4 - a) Desvio padrão da penetração acelerada de íons cloretos conforme o fck; b) Classificação da penetrabilidade de íons cloreto nos concretos estudados.

A partir dos resultados apresentados pelo ensaio de penetração de íons cloretos (Figura 4) é possível observar uma redução dos valores para carga passante, indicando que com a diminuição do fator a/c do concreto, tende a diminuir a capacidade de penetração dos íons cloreto. Isso pode ser analisado na Figura 5, onde consta uma correlação entre o fator a/c dos concretos e a carga passante $(C)$ nos concretos investigados.

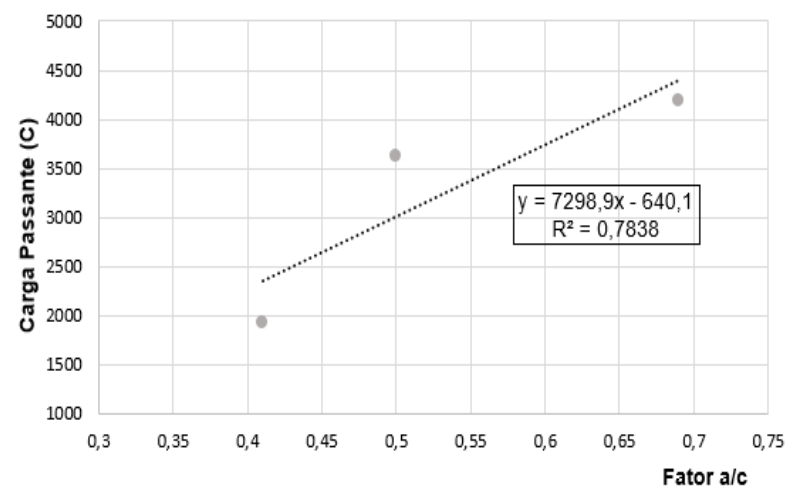

Figura 5 - Correlação entre a fator a/c e a carga passante (C) no ensaio de penetração acelerada de íons cloreto

Constata-se ainda, conforme Figura 4 que para a classe de resistência de $20 \mathrm{MPa}$ foram classificados como alta penetrabilidade. Já para as classes de $30 \mathrm{MPa}$ e 40 MPa houve uma melhoria na penetrabilidade de íons cloretos. Enquanto para a classe de 30 foram classificados como penetrabilidade de íons cloretos moderada, os traços de $40 \mathrm{MPa}$ foram classificados como baixa penetrabilidade. Em estudo realizado por Crauss [17] é possível observar que o tipo de cimento influencia diretamente a capacidade de penetração de íons cloretos no concreto. Sendo o cimento CP V, o mesmo utilizado nessa pesquisa, foi o que apresentou os piores resultados. O melhor desempenho foi alcançado pelos traços executados com cimento Portland Pozolânico (CP IV), devido ao refinamento dos poros do concreto promovido pela cinza volante presente em sua composição. Com isto, a utilização do cimento CP IV, CP II ou ainda, a utilização de adições apresentaria melhores valores, se comparada com obtidos com o cimento CP V. 
DIAS, A. R. O.; AMANCIO, F. A.; LUCAS, S. O.; LIMA, D. A.; CABRAL, A. E. B., AVALIAÇÃO DA PENETRAÇÃO ACELERADA DE ÍONS CLORETOS EM CONCRETOS PRODUZIDOS COM MATERIAIS DA CIDADE DE FORTALEZA. $4^{\circ}$ Simpósio Paranaense de Patologia das Construções (40 SPPC), artigo 4SPPC109, pp. 70 - 79, 2019. DOI: 10.4322/2526-7248.030

Por fim, foi realizado a Análise de Variância a fim de determinar a influência do fck na penetração acelerada de íons cloretos nos concretos estudados. Tais resultados podem ser observados na Tabela 7.

Tabela 7- ANOVA para o ensaio de penetração acelerada de íons cloretos.

\begin{tabular}{ccccccc}
\hline \multicolumn{7}{c}{ Análise de variância } \\
\hline Propriedade & $\mathrm{SQ}$ & $\mathrm{GL}$ & $\mathrm{MQ}$ & $\mathrm{F}$ & Valor- $\mathrm{p}$ & Significância \\
fck & 8334547 & 2 & 4167273 & 450,09 & 0,000000 & $\mathrm{~S}$ \\
Erro & 55553 & 6 & 9259 & & & \\
\hline Legenda: SQ- Soma quadrada; $\mathrm{GL}-$ Grau de liberdade; MQ- Média quadrada; S-
\end{tabular}

Significativo; NS - Não significativo

Com a realização da ANOVA foi possível identificar que a classe de resistência apresenta influência significativa na penetração acelerada de íons cloretos, ao passo que com o aumento do fck do concreto, ocorre uma redução significativa da carga passante $(C)$ no ensaio de penetração acelerada de íons cloretos do concreto.

\section{Considerações Finais}

O presente estudo teve por objetivo avaliar a penetração acelerada de íons cloretos, conforme a ASTM C1202:2012, em concretos de diferentes classes de resistência produzidos com materiais da cidade de Fortaleza. Os concretos de classe de resistência maiores apresentaram menores carga passantes, o que os tornam mais resistentes à penetração de íons cloretos e consequentemente possuem maior durabilidade.

Os concretos de classe de resistência 20MPa, 30MPa e 40MPa apresentaram risco de penetração de íons cloretos em alto, moderado e baixo, respectivamente. A análise estatística, por meio da Análise de Variância demonstrou que o fck do concreto exerce influência significativa na penetração de íons cloretos, sendo os concretos com maiores fck os que apresentam os menores valores de carga passante $(C)$. Isto se deve ao menor fator a/c desses concretos, e consequentemente, menor porosidade.

Por fim, tendo em vista que a deterioração precoce das estruturas de concreto armado provocadas pela corrosão, além de afetar sua estabilidade, gera elevados custos com o reparo. Sugere-se a realização de novos ensaios no intuito de investigar os concretos com outros tipos de cimento, bem como com o uso de adições pozolânicas no intuito de melhorar a durabilidade e aumentar a vida útil desses concretos, em virtude da elevada agressividade do ar da cidade de Fortaleza, devido à alta concentração de íons cloretos no ar.

\section{Agradecimentos}

Os autores agradecem ao Programa de Pós-Graduação em Engenharia Civil: Estruturas e Construção Civil (PEC) e ao Laboratório de Materiais de Construção Civil (LMCC) da Universidade Federal do Ceará - UFC. O presente trabalho foi realizado com apoio da Coordenação de Aperfeiçoamento de Pessoal de Nível Superior - Brasil (CAPES) - Código de Financiamento 001. 
DIAS, A. R. O.; AMANCIO, F. A.; LUCAS, S. O.; LIMA, D. A.; CABRAL, A. E. B., AVALIAÇÃO DA PENETRAÇÃO ACELERADA DE ÍONS CLORETOS EM CONCRETOS PRODUZIDOS COM MATERIAIS DA CIDADE DE FORTALEZA. $4^{\circ}$ Simpósio Paranaense de Patologia das Construções (40 SPPC), artigo 4SPPC109, pp. 70 - 79, 2019. DOI: 10.4322/2526-7248.030

\section{Referências}

[1] MEHTA, P. K.; MONTEIRO, P. J. M. Concreto: Microestrutura, propriedades e materiais. 3aㅡ ed. São Paulo: IBRACON, 2013.

[2] RINCÓN, O. T. Duracon Collaboration. Durability of concrete structures: DURACON, an iberoamerican project. Preliminary results. Building and environment. Vol. 41. pp. 952-962, Julho, 2006.

[3] CABRAL, A. E. B. Avaliação dos sistemas de reparo utilizados em estruturas atacadas por cloretos através da resistência de polarização. (Dissertação de mestrado). Universidade Federal do Rio Grande do Sul, Porto Alegre, 2000.

[4] SHI, XIANMING; XIE, NING.; FORTUNE, KEITH.; GONG, JING. Durability of steel reinforced concrete in chloride environments: An overview. Construction and Building Materials. v.30, pp. 125-138, Maio, 2012.

[5] AïTCIN, P.C. The durability characteristics of High-Performance Concrete: a review. Cement and Concrete Composites, v.25, p.409-420, 2003

[6] TORRES, A. S.Corrosão por cloreto em estruturas de concreto armado: uma meta-análise. (Tese de doutorado). Universidade Federal do Rio Grande do Sul, Porto Alegre, 2011.

[7] PORTELLA, C. S. A. Avaliação da durabilidade de concretos produzidos segundo a ABNT NBR 6118:2007 e expostos aos cloretos disseminados em névoa salina na Praia do Futuro, em Fortaleza-CE. 2013. Dissertação (Mestrado em Engenharia Civil)- Centro de Tecnologia, Programa de Pós-Graduação em Engenharia Civil: Estruturas e Construção Civil, Universidade Federal do Ceará, Fortaleza, 111 p. 2013.

[8] CAMPOS, A. M. R. Estudo da agressividade do ar atmosférico de Fortaleza/CE. 2016. Dissertação (Mestrado em Engenharia Civil: Estruturas e Construção Civil)Centro de Tecnologia, Universidade Federal do Ceará, Fortaleza, 138 p. 2016.

[9] AMERICAN SOCIETY FOR TESTING AND MATERIALS. ASTM C 1202. Standard test method for electrical indication of concrete's ability to resist chloride ion penetration. Philadelphia, 2012.

[10] ISAIA, G.C. Efeito de Misturas Binárias e Ternárias de Pozolanas em Concreto de Elevado Desempenho: um estudo de Surabilidade com Vista à Corrosão da Armadura. 1995. 280f. Tese (Doutorado em Engenharia) - Escola Politécnica da Universidade de São Paulo - EPUSP, São Paulo, 1995.

[11] ASSOCIAÇÃO BRASILEIRA DE NORMAS TÉCNICAS. NBR 15900-1. Água para amassamento do concreto. Requisitos. Rio de Janeiro, 2009.

[12] ASSOCIAÇÃO BRASILEIRA DE NORMAS TÉCNICAS. NBR 7211. Agregados para concreto. Especificação. Rio de Janeiro, 2009. 
DIAS, A. R. O.; AMANCIO, F. A.; LUCAS, S. O.; LIMA, D. A.; CABRAL, A. E. B., AVALIAÇÃO DA PENETRAÇÃO ACELERADA DE ÍONS CLORETOS EM CONCRETOS PRODUZIDOS COM MATERIAIS DA CIDADE DE FORTALEZA. $4^{\circ}$ Simpósio Paranaense de Patologia das Construções (40 SPPC), artigo 4SPPC109, pp. 70 - 79, 2019. DOI: 10.4322/2526-7248.030

[13] ASSOCIAÇÃO BRASILEIRA DE NORMAS TÉCNICAS. NBR NM 67. Determinação da Consistência pelo abatimento do tronco de cone. Rio de Janeiro, 1998.

[14] ASSOCIAÇÃO BRASILEIRA DE NORMAS TÉCNICAS. NBR 5739. Concreto Ensaio de compressão de corpos de prova cilíndricos. Rio de Janeiro, 2018.

[15] ASSOCIAÇÃO BRASILEIRA DE NORMAS TÉCNICAS. NBR 8522Concreto Determinação dos módulos de elasticidade e de deformação à compressão. Rio de Janeiro, 2017.

[16] ASSOCIAÇÃO BRASILEIRA DE NORMAS TÉCNICAS. NBR 9778. Argamassa e concreto endurecidos - Determinação da absorção de água, índice de vazios e massa específica. Rio de Janeiro, 2005.

[17] CRAUS, C. Penetração de cloretos em concretos com diferentes tipos de cimento submetidos a tratamento superficial. 2010. Dissertação (Mestrado em Engenharia Civil) - Programa de Pós-Graduação em Engenharia Civil, Universidade Federal de Santa Maria, Rio Grande do Sul, 100 p. 2010. 